\title{
TRADUCción EMPRESARIAL EN EL MUNDO GLOBALIZADO
}

\section{BUSINESS TRANSLATION IN TODAY'S GLOBAL}

DE HOY

FECHA DE RECEPCIÓN: 14 de enero FECHA DE APROBACIÓN:10 de marzo Pp. 132-147
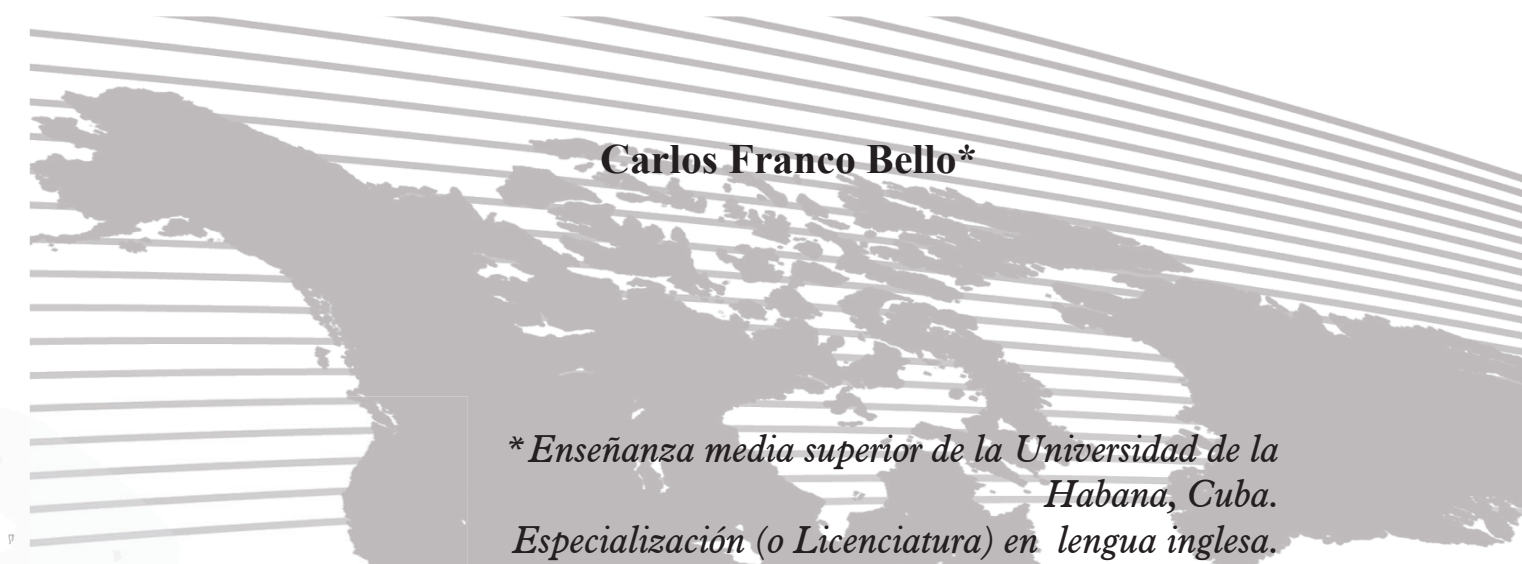
Maestría en gestión de organizaciones, de la Universidad EAN con doble titulación de la Universidad de Québec, Canadá. Maestría en traducción económica, financiera y de negocios internacionales, de la Universidad EAN con - doble titulación de la Universidad Pompeu Fabra, de España.

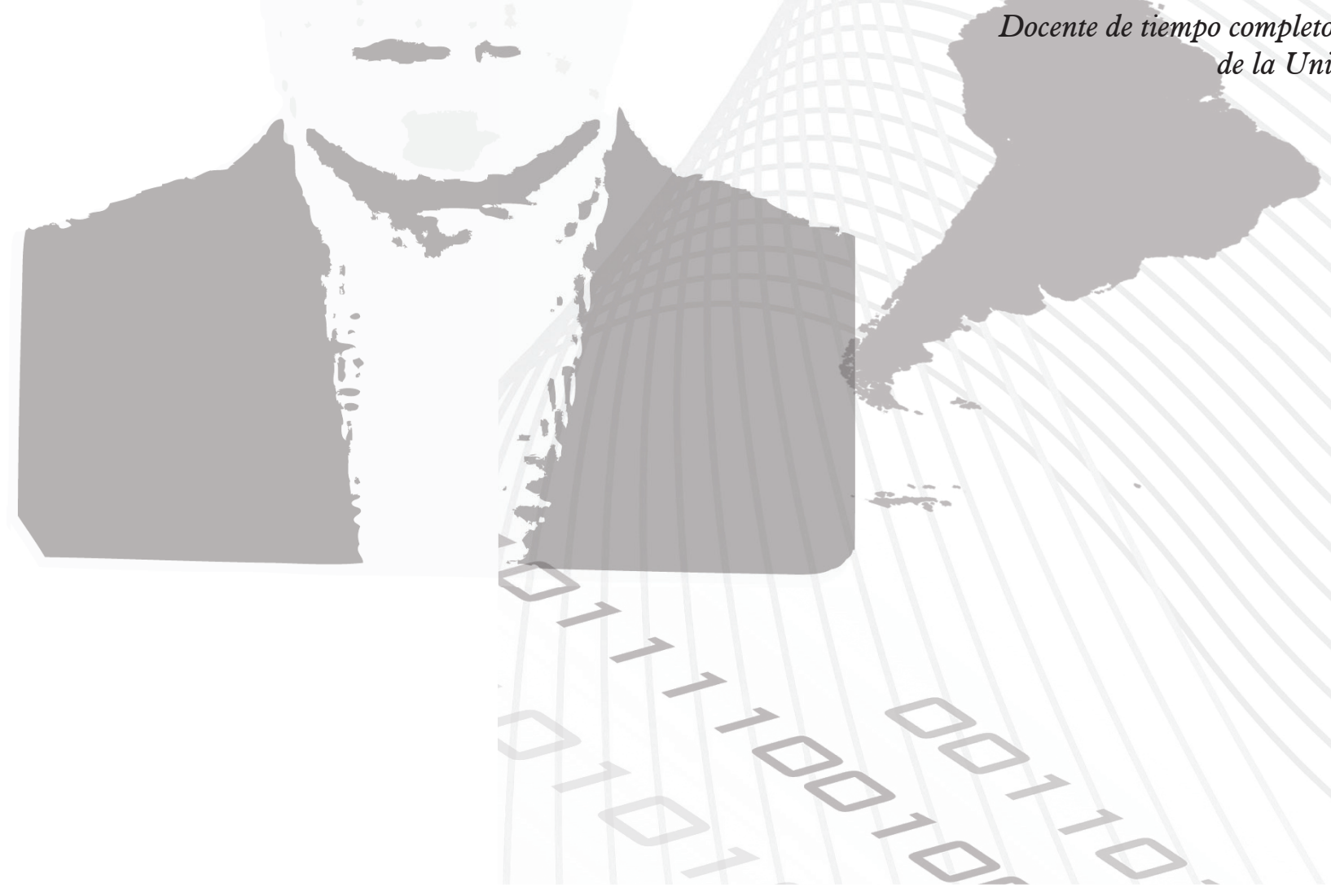




\section{RESUMEN}

Hoy, en un mundo globalizado, el ejercicio de la traducción e interpretación se convierte en una necesidad inminente para el mundo empresarial actual. Por ende, al ser este una práctica empírica y ocasional durante su historia, hoy es necesario que los traductores e intérpretes se hagan conscientes de la necesidad de una profesionalización o especialización no solo en las áreas científico- técnicas, sino también en las áreas económica, financiera y de negocios internacionales, y a su vez la creciente necesidad de que estos profesionales creen su propia empresa con un amplio portafolio de calidad en los campos de la traducción escrita y la interpretación.

Un ejemplo de ello es la experiencia de la Universidad EAN en la profesionalización de los primeros traductores en un programa de doble titulación de Maestría en Traducción Económica, Financiera $y$ de Negocios Internacionales, con la Universidad Pompeu Fabra de Barcelona, España, en un intento de brindar herramientas y estrategias traductoras por parte de expertos nacionales y españoles en ejercicio con un amplio bagaje y experiencia en dichos campos.

\section{ABSTRACT}

Currently, in a globalized world, the professional formation and training of translators and interpreters becomes an outstanding need in the organizational environment. As the exercise of translation and interpretation has always been an empirical and occasional practice through times, it is important that these translators and interpreters become conscious about their growing need to get a formal educational preparation and training, not only in scientific and technical fields, but also in areas such as economy, finances and international business. Therefore, our present world requires that these translation and interpretation professionals create their business plans to set up their own companies to satisfy the demands of other companies and the current market.

An example of these efforts is the experience that Universidad EAN has implemented through a double-diploma master- degree program on scientific and technical, economic, financial and international business translation in coordination with Universidad Pompeu Fabra, in Spain. This postgraduate program aims at providing the necessary translation tools and strategies based on the experience of Spanish and Colombian experts in the fields of translation and interpretation.

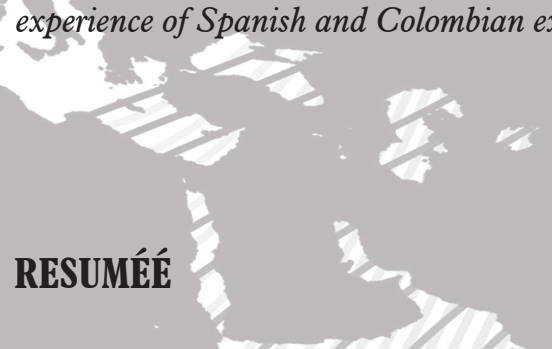

Aujourd'hui, l'exercice de la traduction et de l'interprétation dans un monde globalisé devient un besoin imminent pour le monde des affaires. Cette pratique s'est révélée être occasionnelle et empirique au cours de l'histoire. Il est cependant aujourd'hui nécessaire que les traducteurs et interprètes soient conscients du besoin d'une expertise technique et professionnelle, non seulement dans les domaines scientifiques-, mais aussi dans les domaines des affaires économiques, financières et internationales, et qu'à leur tour ces professionnels puissent créer leur propre entreprise offrant un vaste éventails de possibilités pour le secteur de la traduction écrite et de l'interprétation.

L'université EAN en est un exemple concret. Son expérience dans la formation des premiers traducteurs inscrits à un programme de double diplôme de master en entreprise de traductionéconomique, financière et internationale, en partenariat avec l'Université Pompeu Fabra de Barcelone, Espagne, s'effectue dans le but de fournir des outils et des stratégies de traduction mises en place par des experts nationaux et espagnols exerçant cette profession et ayant une vaste expérience dans ces domaines. $\rightarrow$ Palabras claves

Traducción- traductor- proceso traductor- comunicaciónprofesionalización-

Globalización- interpretaciónintérpretes-corrección de estilo- lengua de partidalengua de llegada- segunda lengua- "freelance translator""staff translator"- sector realsectores económicos-contexto
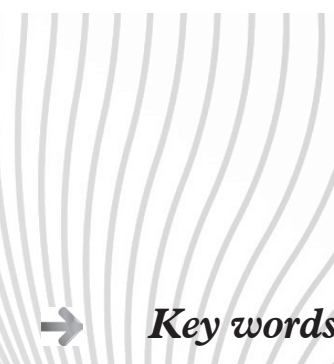

Translation- translatortranslation processcommunicationspecialization-Globalizationinterpretation- interpreterstylistic correction-target language- original languagesecond language-freelance translator-staff translator-real sector- economic sectorscontext 


\section{INTRODUCción}

a traducción ha sido considerada como un oficio de

carácter empírico desde la Edad Media, es por ello que surge esta actividad como producto del auge en el trueque y en el comercio a nivel internacional, así como la expansión colonial hacia nuevos continentes.
Esta actividad crece cada vez más en su demanda actual y ha acompañado al hombre durante toda su evolución histórica, y es hoy cuando dicha actividad juega un papel relevante en las relaciones que se establecen entre las naciones del mundo de hoy y como resultado de la llamada globalización, la cual ha establecido un número infinito de nuevas relaciones económicas y políticas en dicho mundo.

\section{。 \\ 2. LA TRADUCCIÓN Y EL MUNDO GLOBAL}

n el mundo global actual, se hace necesario el crecimiento profesional y la especialización de todos aquellos que tienen que operar en las distintas áreas del conocimiento humano, cuando este se convierte en el arma más preciada del hombre para poder interactuar con su medio. Por consiguiente, la traducción o proceso traductor no escapa de esta necesidad y hoy se constata

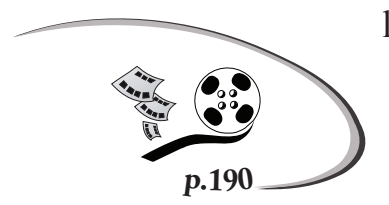

la gran necesidad de preparar traductores e intérpretes ya sea mediante carreras profesionalizantes, o mediante cursos especializados y de postgrado, aunque cabe resaltar que los mismos no se encuentran ofertados en mayor cuantía en el mercado educativo actual en Colombia y Latinoamérica.

Existen pocos estudios acerca de este campo de la traducción y la interpretación, aunque en España se evidencian desarrollos muy importantes en los últimos tiempos enfocados en la traducción científico- técnica. Sin embargo, en Colombia, después de realizar El Encuentro Nacional de Traductores, organizado por el Colegio Colombiano de Traductores y la Universidad EAN, realizado en octubre de 2006, se comienza a explorar la necesidad de profesionalizar y especializar a traductores e intérpretes en los campos económico, financiero y de negocios internacionales.

Por otro lado, existen en Colombia asociaciones de traductores e intérpretes que realizan una ardua labor por agrupar a éstos y dar unos estándares de calidad al ejercicio de la traducción e interpretación. Entre estas asociaciones encontramos el Colegio Colombiano de

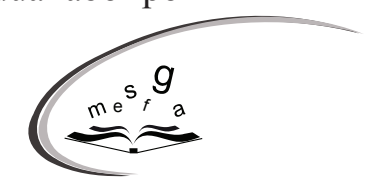
Traductores, la Asociación Colombiana de Traductores e Intérpretes, el ICONTEC, entre otras. 
En Colombia actualmente, el traductor e intérprete es una persona que trabaja de forma independiente generalmente, realizando trabajos para clientes particulares y en otras ocasiones para empresas en los momentos que estas requieran dichos servicios de manera ocasional. Estos traductores e intérpretes realizan este oficio por vocación y no por formación profesional, siendo generalmente personas que han aprendido una segunda lengua, básicamente el inglés, y por lo menos demuestran buen dominio de su lengua materna, el español, y de la segunda lengua, así como conocimientos generales de sus culturas, producto de algunas experiencias de contactos e inmersiones en las mismas. En su mayoría, los traductores e intérpretes empíricos se especializan en la traducción e interpretación en solo una de las distintas áreas del conocimiento humano, y en su mayoría realizan trabajos del corte científico- técnico.

En este sentido, la práctica empírica del ejercicio de la traducción consiste en traspasar o transpolar palabras de una lengua de partida a otra lengua de llegada, sin tener en cuenta ni la parte lingüística ni tampoco los elementos culturales de dicha lengua de llegada. Cuando esta actividad se realiza de esta manera, la traducción carece de un sentido verdaderamente profesional y de calidad óptima como producto final. Es muy importante destacar que el traductor necesita dar un contexto apropiado a las ideas o conocimientos por traducir en cualquier área al traspasar éstos a una segunda lengua.

Otro elemento importante a resaltar, es el proyecto desarrollado por un grupo de docentes de la carrera de Lenguas Modernas de la Universidad EAN: Traducción para Empresas Contemporáneas, del cual se derivó un texto llamado Babel en las Empresas Colombianas- Una Mirada Actual de la Traducción, 2008. En este trabajo de investigación y como resultado en el texto escrito como producto final se hace una descripción detallada del estado de la traducción y la interpretación así como del papel del traductor e intérprete en el contexto Latinoamericano, y particularmente en Colombia.

\section{ALGUNAS REFLEXIONES SOBRE EL PROCESO TRADUCTOR ACTUAL}

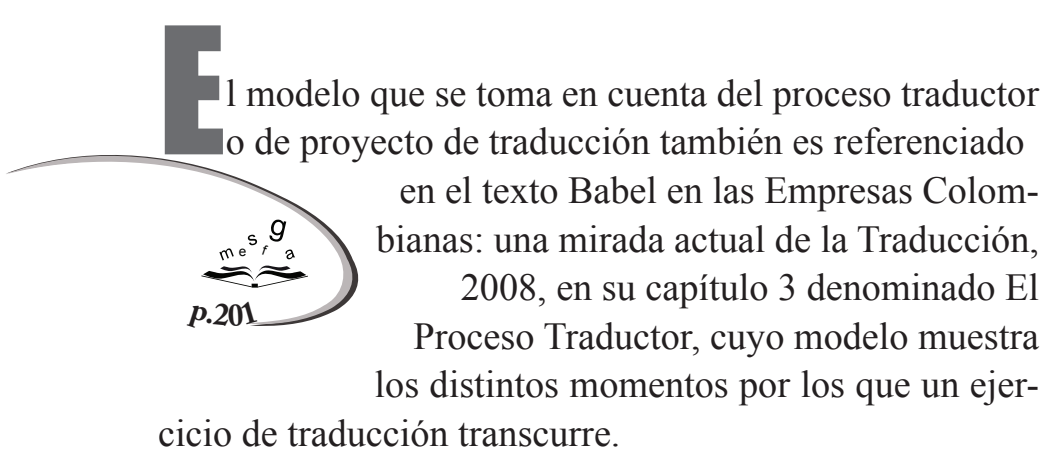

Dicho modelo contiene las siguientes etapas:
- Abordaje del texto.

- Reunión de fuentes.

- Preparación del vocabulario.

- Traducción del texto.

- Revisión formal.

- Lectura final.

- Las dudas.

- La entrega.

- El archivo. 
Figura 2. Proceso de traducción

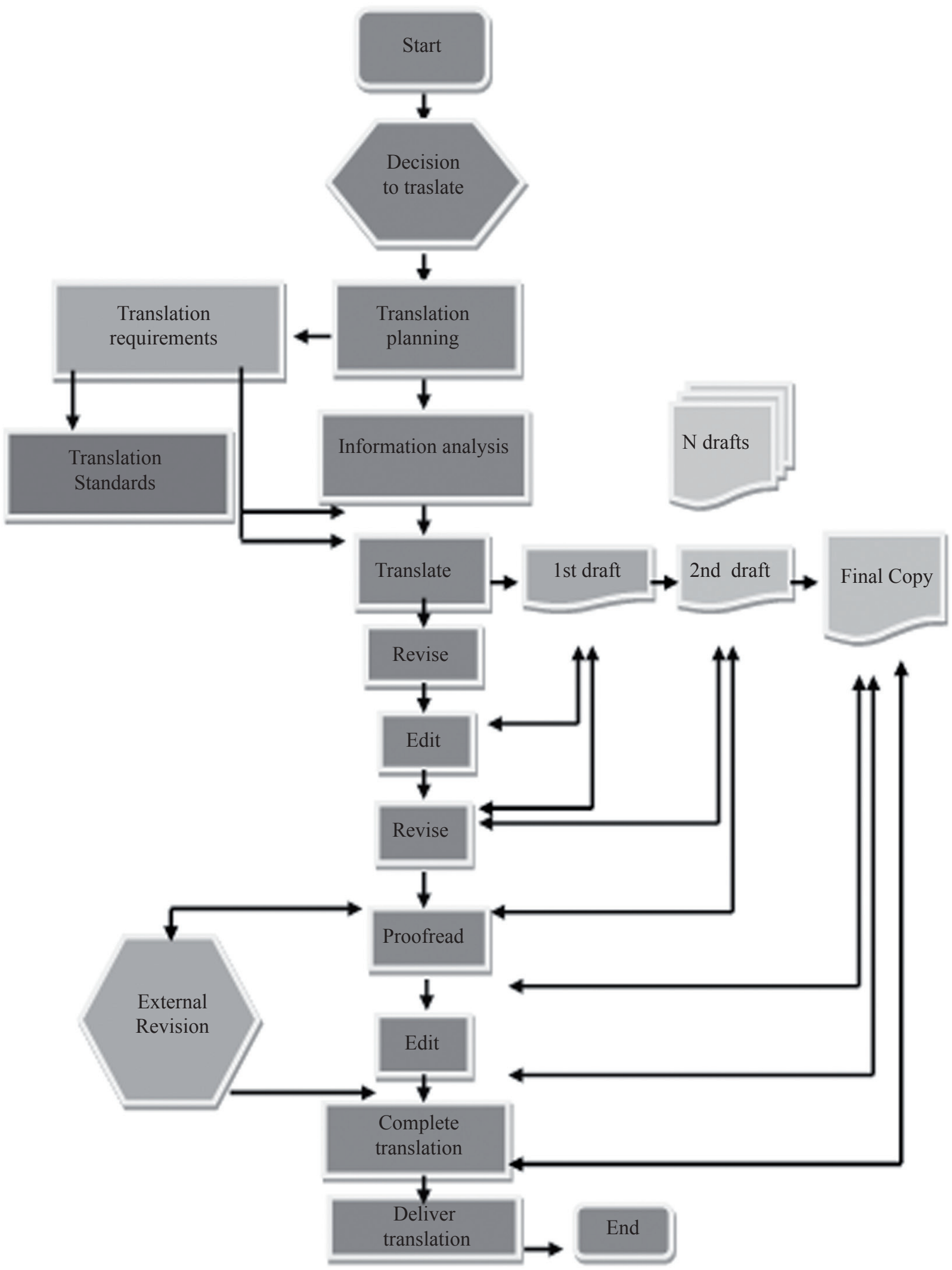

Fuente. Darwish, (2008) 
El llamado proceso traductor planteado originalmente por Ali Darwish en su texto Towards a theory of Constraints in Translation, 2008, describe este proceso como un proyecto de traducción basado en diferentes etapas, las cuales se llevan a cabo desde el inicio del proyecto hasta su entrega final del producto.

En un comienzo, este trabajo se define a partir de la necesidad real de un cliente, quien contacta a la empresa o al traductor directamente, y le plantea el trabajo de traducción a realizar en el área correspondiente. De aquí, el traductor acepta su realización, si el tema está dentro de su campo de acción temático y experiencia. En este momento, es cuando se realiza la planeación del proyecto, determinando el tiempo de entrega, así como los requisitos o detalles que este proyecto necesita a la hora de ser entregado al cliente. Luego de este paso, el traductor establece unos estándares que debe tomar en cuenta durante todo el proceso para realizar el trabajo de traducción satisfactoriamente.

En este punto, es cuando empieza el traductor realmente su ejercicio de traducción, realizando la lectura del documento a traducir y analizando la información correspondiente mediante la exploración del tema. Una vez realizado este preámbulo, el traductor comienza a traducir, apoyándose en herramientas tecnológicas, diccionarios especializados, glosarios de terminología o memorias de traducción, consulta de textos paralelos de traducciones realizadas anteriormente sobre el tema y que el propio cliente posea. En esta etapa, el traductor redacta tantos borradores como sean necesarios, consultando al cliente en ocasiones sobre temas no claros. Sobre cada borrador se realiza un trabajo de revisión y de edición de una nueva versión.

Finalmente, una vez el traductor tenga la versión final del trabajo, este lo entrega a un par o colega traductor de su confianza y que posea experiencia en el tema trabajado con el fin de que este haga una evaluación externa del mismo. Cuando este par entrega su retroalimentación al traductor, este realiza la edición final del documento y procede a realizar la entrega del mismo al cliente para proceder a cerrar el proyecto mediante el pago del trabajo realizado entregado en tiempo, y según los acuerdos concertados desde el comienzo del proyecto entre el traductor y el cliente. De esta forma, el proyecto finaliza satisfactoriamente.

Por otra parte cabe resaltar el estado de los servicios de traducción e interpretación que se ofrecen hoy en el mercado, clasifican al traductor e intérprete (según SamuelsonBrown, 2004) como freelance, el cual trabaja desde su casa o su propia oficina, o como otra opción aparece el staff translator, el cual hace parte de un departamento en una empresa u organización. En ambos casos, y como se manifestó anteriormente, esos

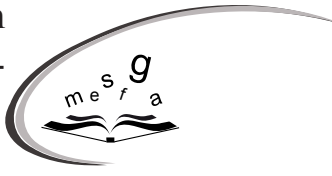
traductores son contratados generalmente no por su preparación profesional o experiencia, sino por su conocimiento totalmente empírico en el manejo de una lengua de llegada, como resultado de cursos tomados en otros países, o de experiencias de contactos o inmersión en la cultura de esa lengua.

En el preámbulo brindado anteriormente, evidenciamos que los servicios de traducción e interpretación en idiomas español e inglés, y en los campos económico, financiero y de negocios internacionales y científico- técnico, son escasos, y se tornan aún más escasos cuando son evaluados bajo estándares normas de calidad existentes actualmente.

De la misma manera, los lugares que ofrecen servicios de corrección de estilo de documentos traducidos son casi inexistentes. Este tipo de actividad la desarrolla también una persona como freelance, la cual ha tenido formación como profesor de español e inglés. Por consecuencia, estos servicios tampoco existen con un carácter competitivo y de alta calidad en el mercado actual, en un contexto que exige cada vez más el uso de documentos para legalizar estados de las organizaciones, así como para realizar actividades de intercambio comercial y negociaciones.

Las traducciones en Colombia son generalmente realizadas por traductores independientes que no tienen formación profesional en traducción y que forman parte del sector, más por vocación que por formación (Clavijo, 2008). 
Gráfica 2. Traductores independientes o en empresa o en agencia

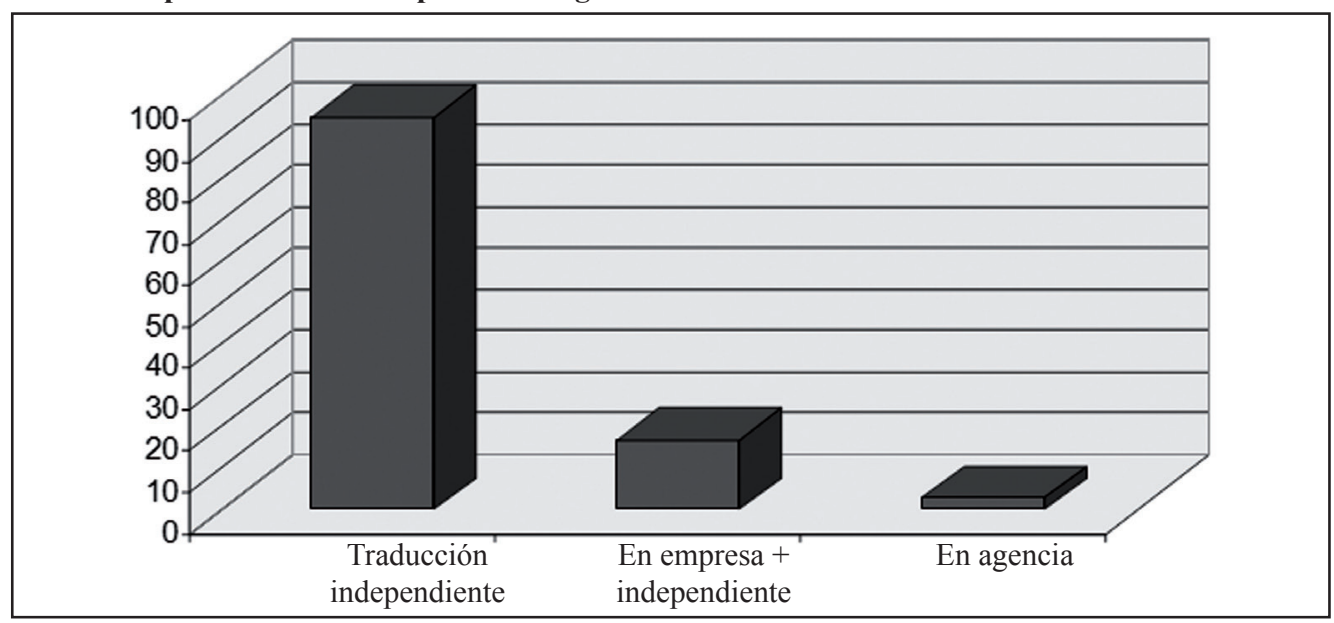

Fuente. Clavijo, et al (2008)

E1 95\% de los traductores en Colombia trabaja de manera independiente.

Gráfica 3. Lengua materna de quienes ejercen la traducción en Colombia

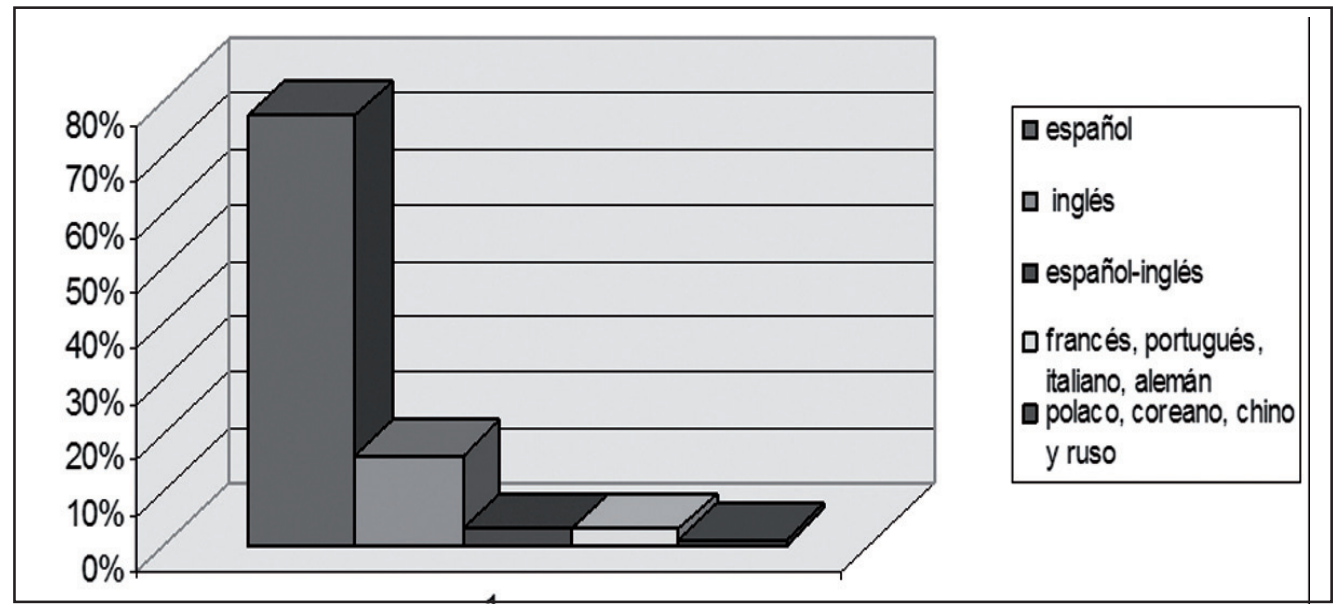

Fuente. Clavijo, et al (2008)

Como se puede observar en la gráfica, el español es la lengua materna del $76 \%$ de los traductores en Colombia, y el inglés es la lengua materna del $16 \%$ de estos.

Gráfica 4. Traductores e intérpretes oficiales y no oficiales

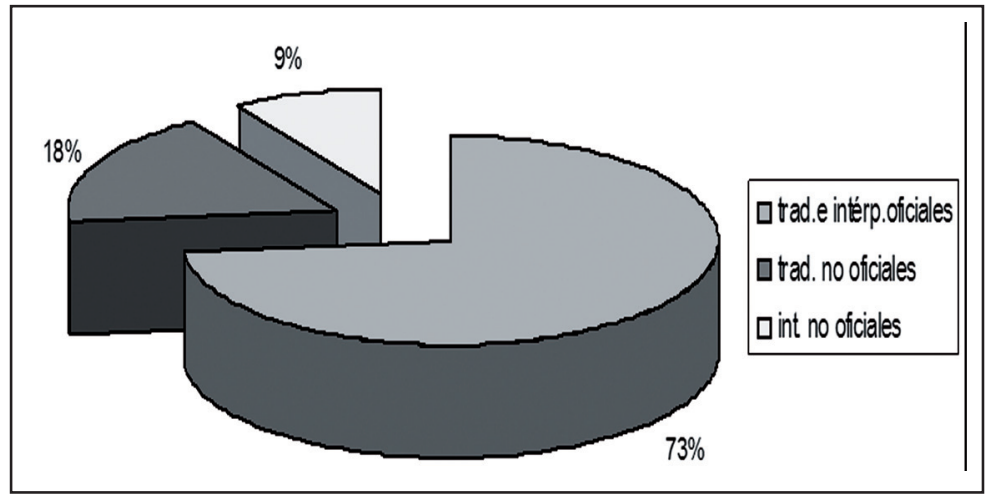

Fuente. Clavijo, et al (2008) 
E1 73\% de los traductores e intérpretes en Colombia son oficiales. En Colombia, el ser traductor oficial no representa necesariamente el tener una formación académica en el campo de la traducción. El traductor oficial en Colombia es la persona que ha aprobado el examen oficial de traducción y que por lo tanto está autorizada a traducir documentos oficiales.

Gráfica 5. Nivel de formación de quienes traducen

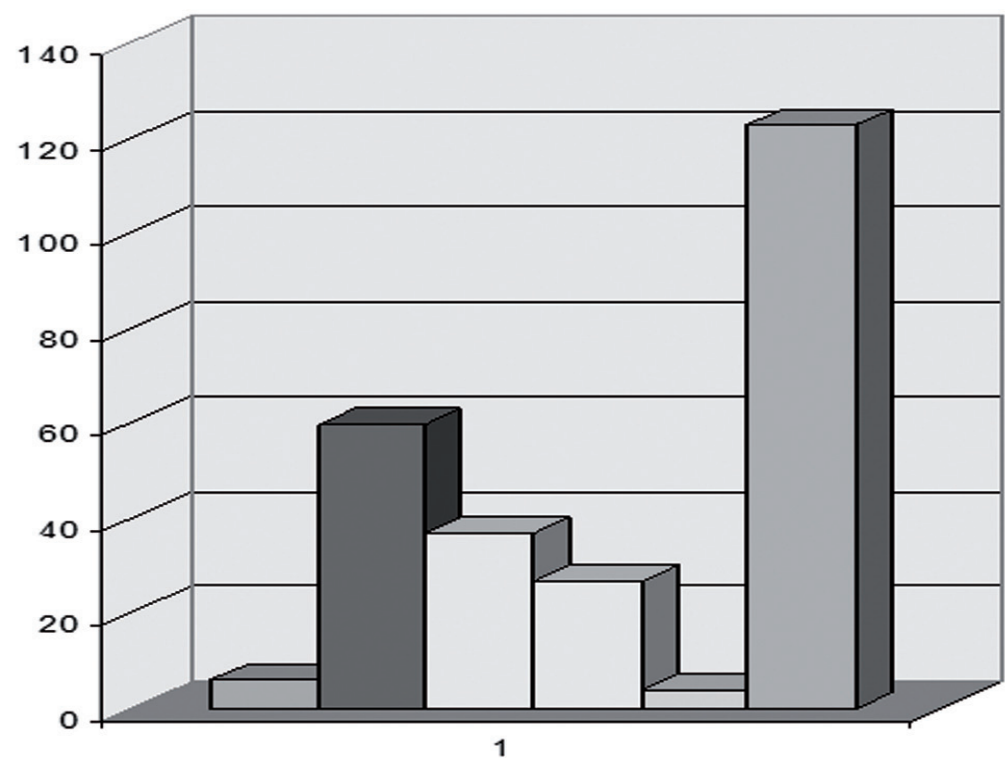

$\square$ Técnico

$\square$ Profes ional

口 Es pecialis ta

口 Magís ter

$\square$ Doctor

$\square$ Edn no formal

Fuente. Clavijo, et al (2008)

Como se puede observar, el 50\% de estos tiene pregrado, sin embargo, una gran mayoría tiene cursos de educación informal (Clavijo, et al, 2008).
En conclusión se puede decir que los traductores en Colombia generalmente son personas que trabajan independientemente, por su propia cuenta, sin equipo de trabajo, y en su mayoría tienen como lengua materna el español, que generalmente no implica que posean competencias traductoras, más allá del conocimiento de otra lengua.

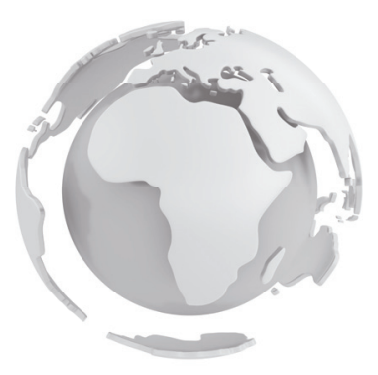




\section{UNA EXPERIENCIA CONCRETA EN EL CONTEXTO COLOMBIANO}

n el caso de la Universidad EAN, y desde su oferta

de postgrados, se realizó un estudio cuidadoso del mercado en el tema del ejercicio y demanda de la traducción, y como resultado del mismo se diseñó y se puso en funcionamiento un programa de Maestría en Traducción Económica, Financiera y de Negocios Internacionales con doble titulación con la Universidad Pompeu Fabra, de Barcelona, España, donde se combinan el ejercicio de la traducción científico- técnica (experiencia española) con el ejercicio de la traducción empresarial enfocada en las áreas económica, financiera y de negocios internacionales, con una futura extensión a la ciudad de Barranquilla, en convenio con la Universidad Simón Bolívar.

Cabe mencionar que el programa de Lenguas Modernas de la Universidad EAN, en su modalidad de pregrado, estimula en alto grado la formación de profesionales con enfoque en traducción económica, financiera y de negocios internacionales para satisfacer las necesidades que las empresas globales de hoy tienen para poder operar e interactuar entre ellas. En dicho programa, los estudiantes adquieren herramientas suficientes tanto en el campo específico de la traducción empresarial como elementos complementarios de tecnologías que complementan el ejercicio de la traducción aplicada a la empresa.

Asimismo, la Universidad EAN ha realizado un número significativo de diplomados enfocados en la traducción empresarial dirigidos a traductores e intérpretes empíricos en ejercicio y dictados por profesionales altamente reconocidos en dicho ejercicio en Colombia, con el fin de ayudar a fortalecer el perfil ocupacional de los mismos, dada la carencia real de traductores profesionalizados y especializados en los campos económico, financiero y de negocios internacionales.

Por otro lado, resulta satisfactorio mencionar el trabajo realizado desde los procesos de práctica profesional y de proyectos de grado. Cada vez se ha mejorado el nivel de preparación y seguimientos mediante tutorías personalizadas dirigidas a los estudiantes de pregrado en la carrera de Lenguas Modernas de la Universidad EAN. En ambos procesos, los estudiantes han sido reconocidos por los proyectos realizados con un alto nivel de respuesta a solucionar problemas reales de la empresa en el área de la comunicación organizacional, y más específico en los procesos de traducción y tecnologías aplicadas.

En el caso de esos trabajos de grado, un gran número de ellos han sido enfocados en creación de empresas o agencias de traducción, el cual se ha incrementado considerablemente, demostrando a su vez que este ejercicio es parte fundamental de la operación y funcionamiento de las empresas de hoy. Para dichos estudios se han realizados análisis concienzudos aplicando instrumentos en empresas del sector real, tales como encuestas y estudios estadísticos, diagnósticos, intervenciones, entrevistas, acompañamientos, participación directa en procesos internos de las empresas, entre otros, los cuales muestran una necesidad cada vez más creciente de profesionales especializados en el tema de la comunicación y la traducción, pero a su vez muestran como resultado el desconocimiento parcial o total por parte del cuerpo directivo de las empresas para reconocer el proceso traductor y el papel del traductor en el sostenimiento y crecimiento de la empresa global de hoy. 


\section{UN ESTUDIO CONCRETO EN EL CAMPO DE LA TRADUCcIóN}

-EXPERIENCIA PERSONAL

1

ecientemente, y como parte del estudio que he realizado en la Maestría en Traducción Económica, Financiera y de Negocios Internacionales, he realizado un estudio de este mercado para mi trabajo de grado ó tesis de grado en creación de empresa, específicamente la creación de una agencia de traducción, para el cual tomé una muestra de 26 empresas colombianas de tamaño pequeño, mediano y grande, y de sectores económicos diferentes tales como servicios terciarios, farmacéuticos, aeronáutica, autopartes, editorial, diplomacia, minería, petróleo, ONG, publicidad, tecnológico, bebidas y alimentos, salud, construcción, entre otros, mostrando el papel determinante de los procesos de comunicación organizacional, específicamente en el campo de servicios de traducción para la empresa.

En este sondeo realizado en empresas colombianas, se evidencian aspectos relacionados con las áreas específicas de la traducción e interpretación y la corrección de estilo que resultan sorprendentes.

En primer lugar, el $54 \%$ de estas empresas estudiadas manifiesta que no existe una dirección o departamento de comunicaciones que administre y regule dichos procesos.

Gráfica 6. Porcentaje de empresas con dependencias de información v comunicación

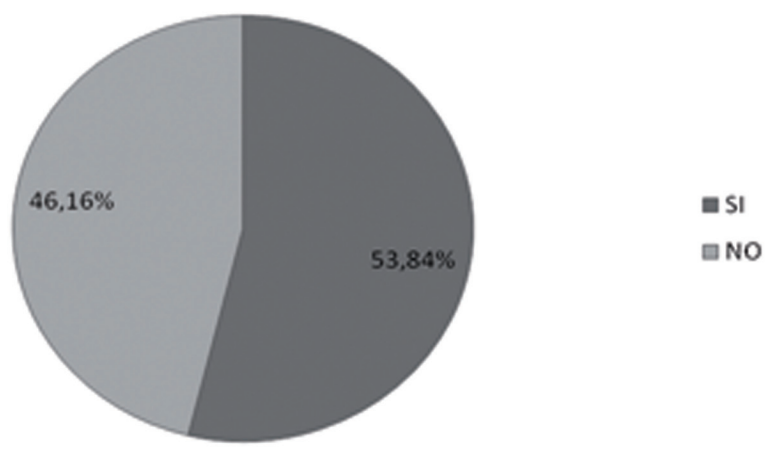

Fuente. El autor

Segundo, el $58 \%$ plantea que sus empresas conciben la español y requieren de servicios de interpretación en corrección de estilo en documentos escritos en idioma reuniones de negocios o eventos.

Gráfica 7. Requerimiento de corrección de estilo de documentos en español

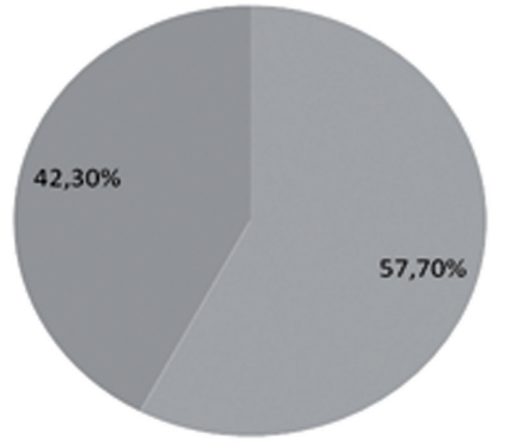

$=\mathrm{SI}$

\pm NO

Fuente. EI autor 
Sin embargo, todas estas empresas analizadas, el $100 \%$, poseen una gran demanda en servicios de traducción escrita en actividades tales como lanzamiento de nuevos productos, negociaciones, redacción de documentos legales, solicitudes, compras, publicaciones, diseño de catálogos, solicitudes de clientes, exportaciones, visitas de extranjeros, entre otros. El $32 \%$ manifiesta requerir dichos servicios en negociaciones y contactos internacionales. Un $12 \%$ revela realizar traducciones de folletos, catálogos, e incluso charlas. Por otros lado, un $4 \%$ afirma que realizan traducciones de páginas web y libros, documentos relacionados con exportaciones, cotizaciones, normas internacionales, capacitaciones, entre otros.

Gráfica 8. Ocasiones en que se requieren servicios de traducción

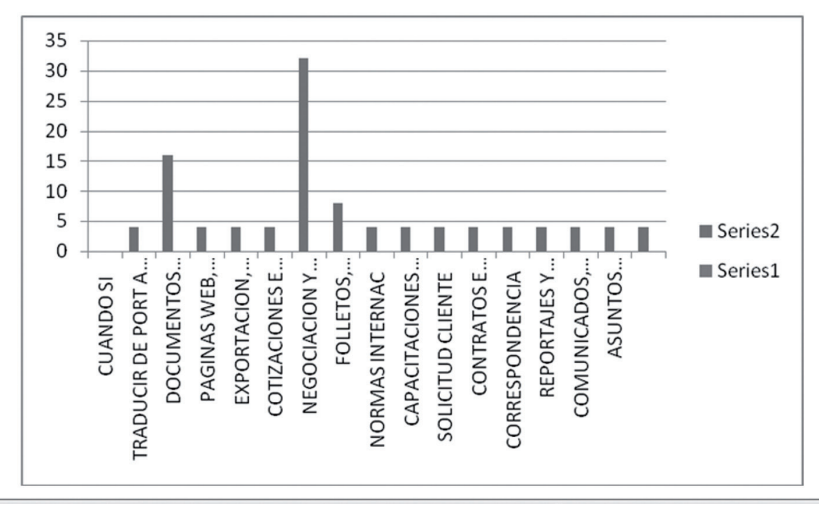

Fuente. El autor

En tercer lugar, las personas que realizan el trabajo de traducción y la corrección de estilo, 17 de 26 empresas dicen que poseen personal interno de esta con conocimientos de un segundo idioma; 14 dijeron que poseen un traductor freelance externo o independiente que las empresas contratan; y sólo 5 empresas manifiestan que contratan los servicios de una empresa de traducción externa.

\section{Gráfica 9. Encargados de la traducción en las empresas}

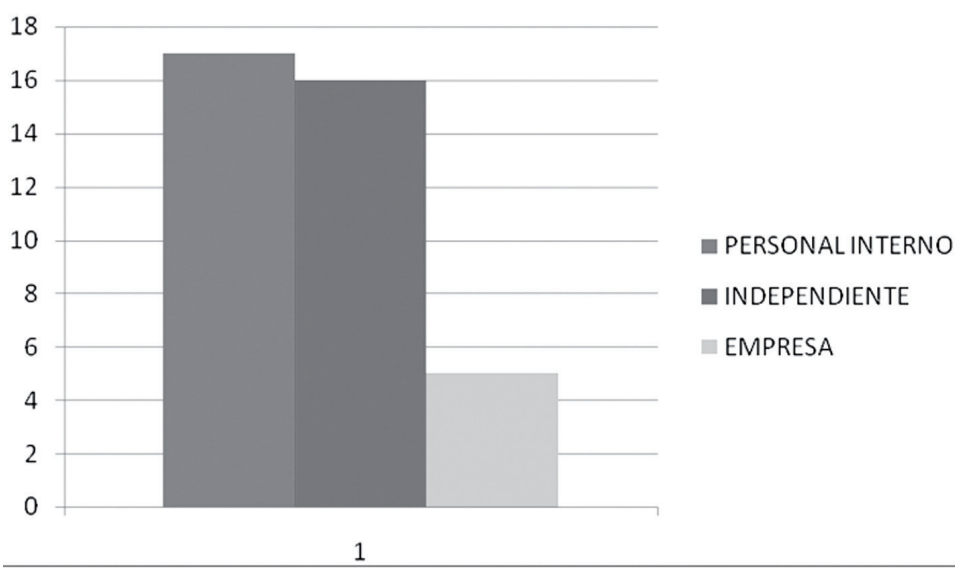

Fuente. El autor

Cuarto, el nivel de satisfacción de estas empresas con respecto al producto final de traducción y corrección de estilo es que el $16 \%$ plantea estar muy satisfechas, el $19 \%$ se encuentra poco satisfechas y el $4 \%$ manifiesta estar nada satisfechas, declarando que las personas que realizan dichas traducciones no son especializados en campos específicos y en otras ocasiones hay problemas con el tiempo de entrega del producto final. 
Gráfica 10. Grado de satisfacción con los servicios de traducción recibidos

\section{Fuente. El autor}

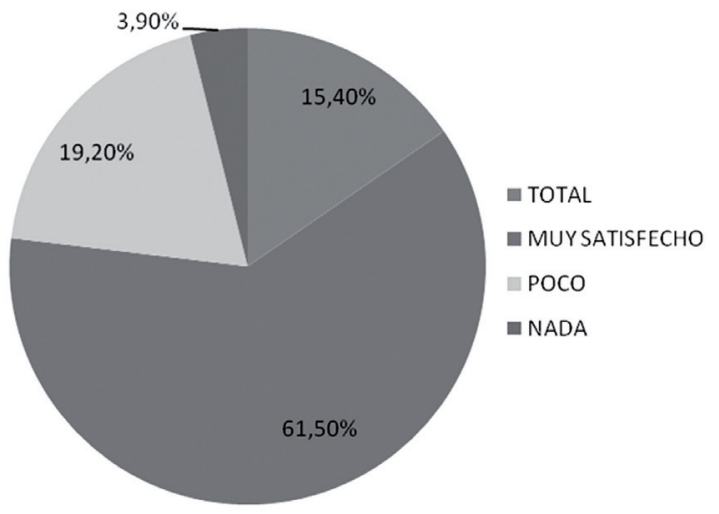

Al analizar, las empresas que utilizan la corrección de estilo de documentos en español, éstas muestran que los aspectos de precio, calidad y rapidez en la entrega son fundamentales, mientras que los aspectos de experiencia del corrector y comentarios recibidos del corrector de estilo o por parte de la propia empresa no son relevantes. De la misma forma, al evaluar el ejercicio de la traducción escrita y su producto final, los aspectos de precio, calidad y rapidez en la entrega son muy significativos, pero la experiencia del traductor y los comentarios recibidos del corrector de estilo o por la propia empresa no son tomados en cuenta nuevamente.

Gráfica 11. Factores que se tienen en cuenta para contratar un corrector de estilo

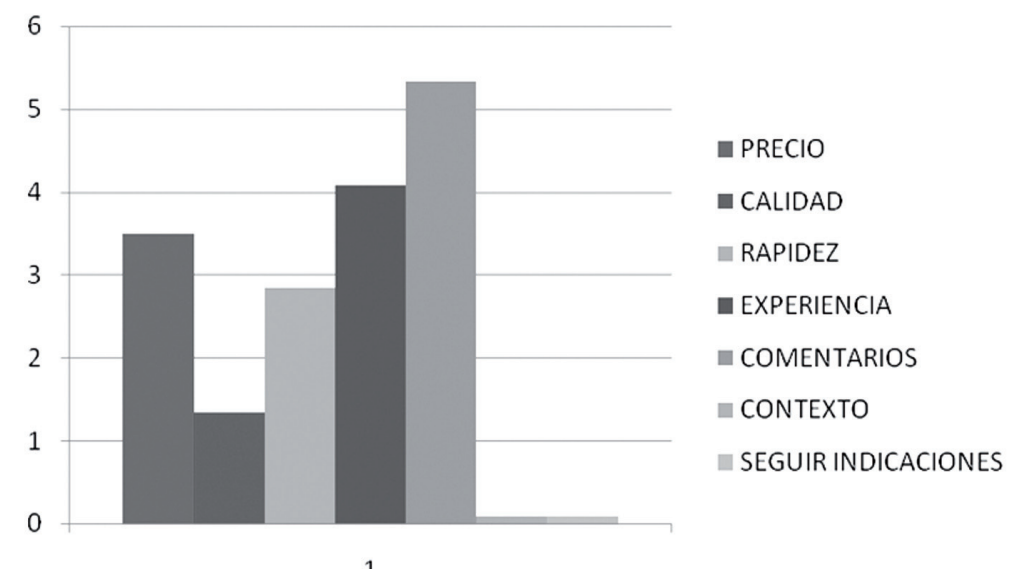

Fuente. El autor

Gráfica 12. Factores que se tienen en cuenta para contratar un traductor

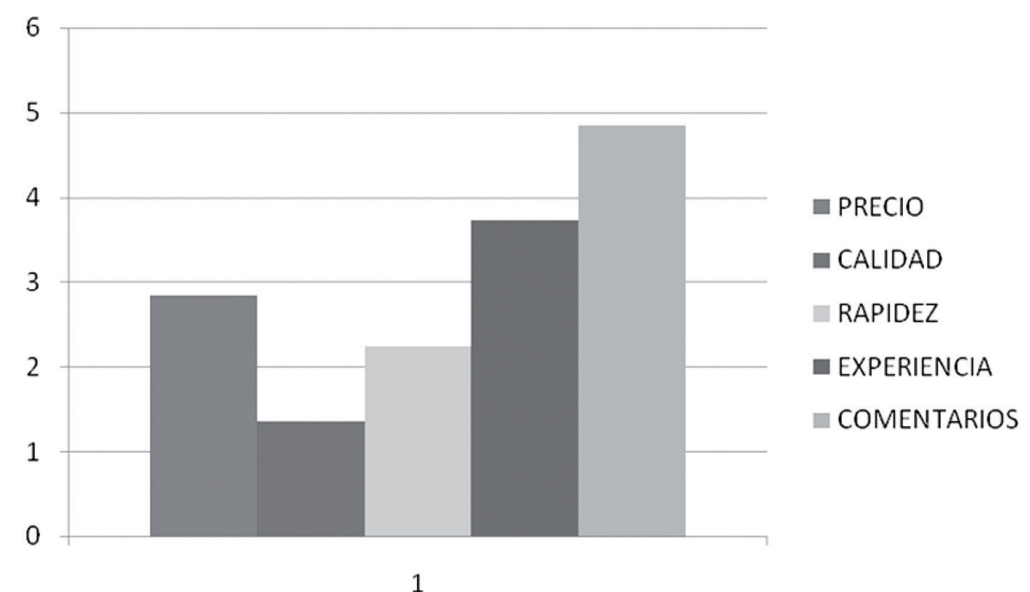

Fuente. El autor 
En el caso de la frecuencia con que se requieren los servicios de corrección de estilo y de traducción escrita en las empresas, el estudio arrojó un resultado de que el $7 \%$ manifiesta que requieren estos servicios menos de una vez por mes; por otro lado el $19 \%$ afirma utilizar estos servicios una vez por mes; el $35 \%$ dice utilizar los servicios de 2 a 3 veces por mes; el $4 \%$ manifiesta utilizarlos entre 3 o 4 veces por mes; y un $35 \%$ utiliza estos servicios de traducción y corrección de estilo 5 o más veces por mes.
Por otro lado, considerando el volumen de material a traducir, el $27 \%$ dice que traduce de 2 a 5 hojas por mes; el $23 \%$ afirma traducir de 6 a 10 hojas por mes; el $15 \%$ traduce de 11 a 20 hojas por mes; y un $35 \%$ menciona que traduce más de 20 hojas por mes. En contraste, el $42 \%$ de estas empresas encuestadas manifiesta que el $100 \%$ de los documentos traducidos pasan por un proceso de corrección de estilo.

Gráfica 13. Número de veces al mes que se usan servicios de traducción

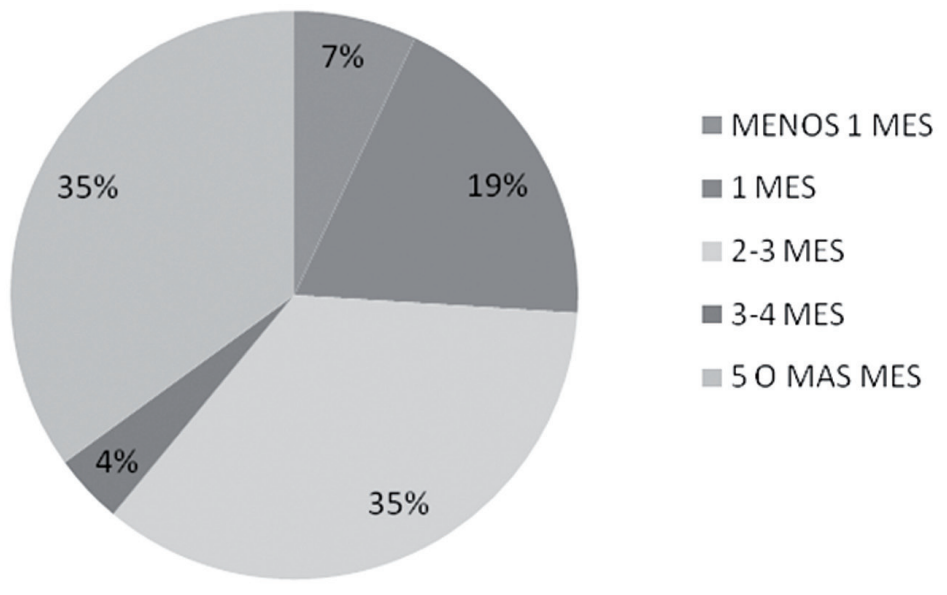

Fuente. El autor

Finalmente, se puede evidenciar que el $50 \%$ de las empresas estudiadas arroja la necesidad de utilizar los servicios de corrección de estilo e interpretación. Además, el $100 \%$ de estas empresas afirma que tienen necesidad de servicios de traducción de documentos escritos, principalmente en negociaciones, cuando de necesita traducir documentos oficiales y catálogos de la empresa. Estas empresas utilizan fundamentalmente personal interno de la empresa para traducir y corregir documentos. Adicionalmente, este estudio muestra que los factores más importantes al contratar servicios de traducción y corrección de estilo son la calidad, seguido de la rapidez y luego el precio del servicio prestado. En las mismas, estos servicios se realizan en un 70 $\%$ de 3 a más de 5 veces por mes, y en una misma proporción se traducen de 5 a 20 hojas por mes. Por otro lado, casi un $50 \%$ de las empresas encuestadas someten los documentos traducidos por un proceso de corrección de estilo.

Gráfica 14. Promedio de hojas traducidas al mes

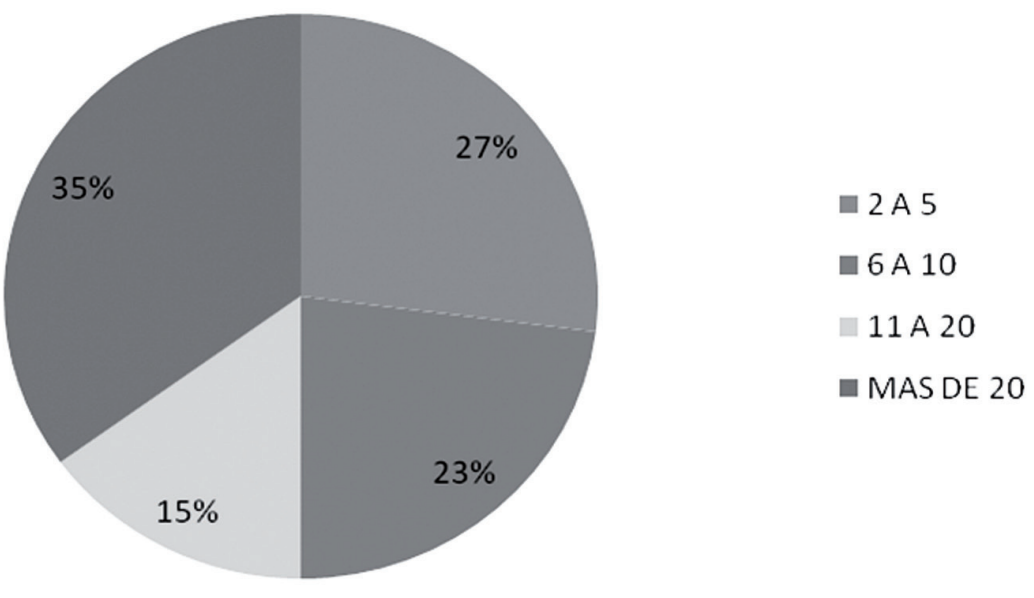


Estos resultados obtenidos del estudio de mercado justifican contundentemente la existencia de un mercado potencial cada vez más creciente que requiere de los servicios de traducción escrita y de corrección de estilo de documentos empresariales.

Gráfica 15. Porcentaje de documentos traducidos y con corrección de estilo

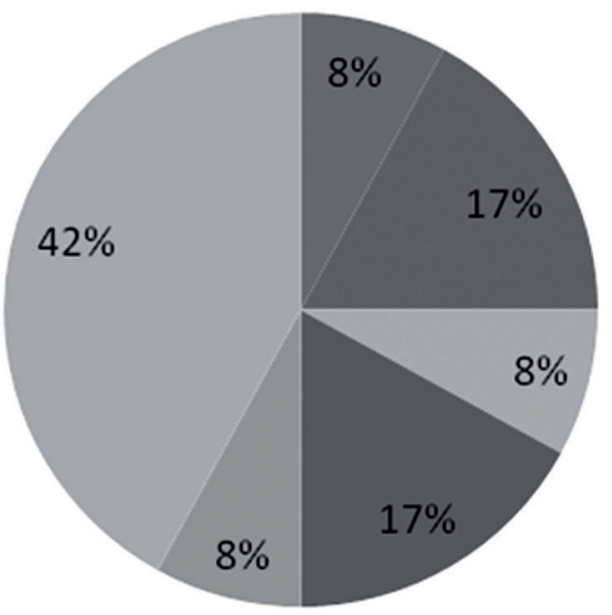

Fuente. El autor

P esulta inminente el trabajo de profesionalización y especialización de traductores e intérpretes en campos especializados que no solo pertenezcan a las áreas científico- técnicas, sino también en las áreas económica, financiera y de negocios internacionales que se encuentran ligadas al entorno empresarial de hoy.

Por otro lado, los esfuerzos como nación en torno a dicho ejercicio empírico, que muestra cada vez una demanda creciente en el mercado de las relaciones globales en el mundo actual, deben estar dirigidos a la profesionalización y especialización del ejercicio de la traducción e interpretación en campos especializados que sobrepasen las áreas científico- técnicas. En este orden de ideas cabe resaltar el trabajo que ha realizado la Universidad EAN en el contexto colombiano para poder impulsar dichos esfuerzos mediante su portafolio de programas de calidad en pregrado, postgrado y extensión, dirigidos a profesionalizar y especializar a los traductores e intérpretes en campos más allá de las áreas tradicionalmente cubiertas por dichos ejercicios.

Finalmente, es imprescindible que las empresas del sector real se concienticen con dichas necesidades para que junto a los esfuerzos por parte del sistema de educación actual, contribuyan al logro de dichos objetivos. 


\section{Bibliografía}

Campbell, S. (1998). Translation into the Second Language. Longman, London.

Clavijo, S. et al, (2008). Babel en las Empresas Colombianas: una mirada actual de la traducción. Universidad EAN, Bogotá.

Darwish, A. (2008). Towards a Theory of Constraints in Translation. En http:www.at-turjuman.com.

Izquierdo, R. (1989). Técnicas de la Traducción y de la Interpretación. Departamento de Textos y Materiales Didácticos. Universidad de la Habana

Neubert, A. (1985). Text and Translation. Leipzig.

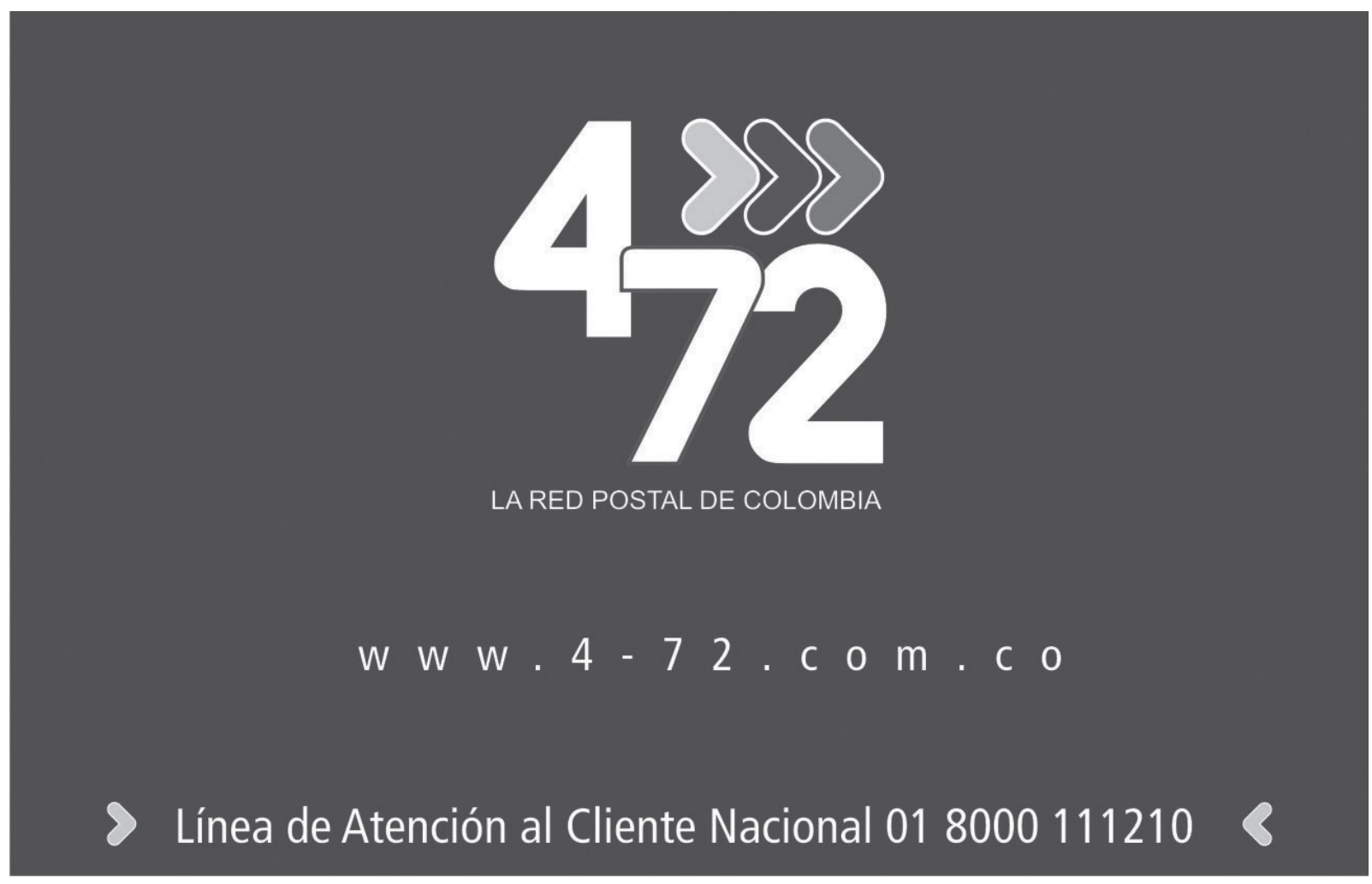




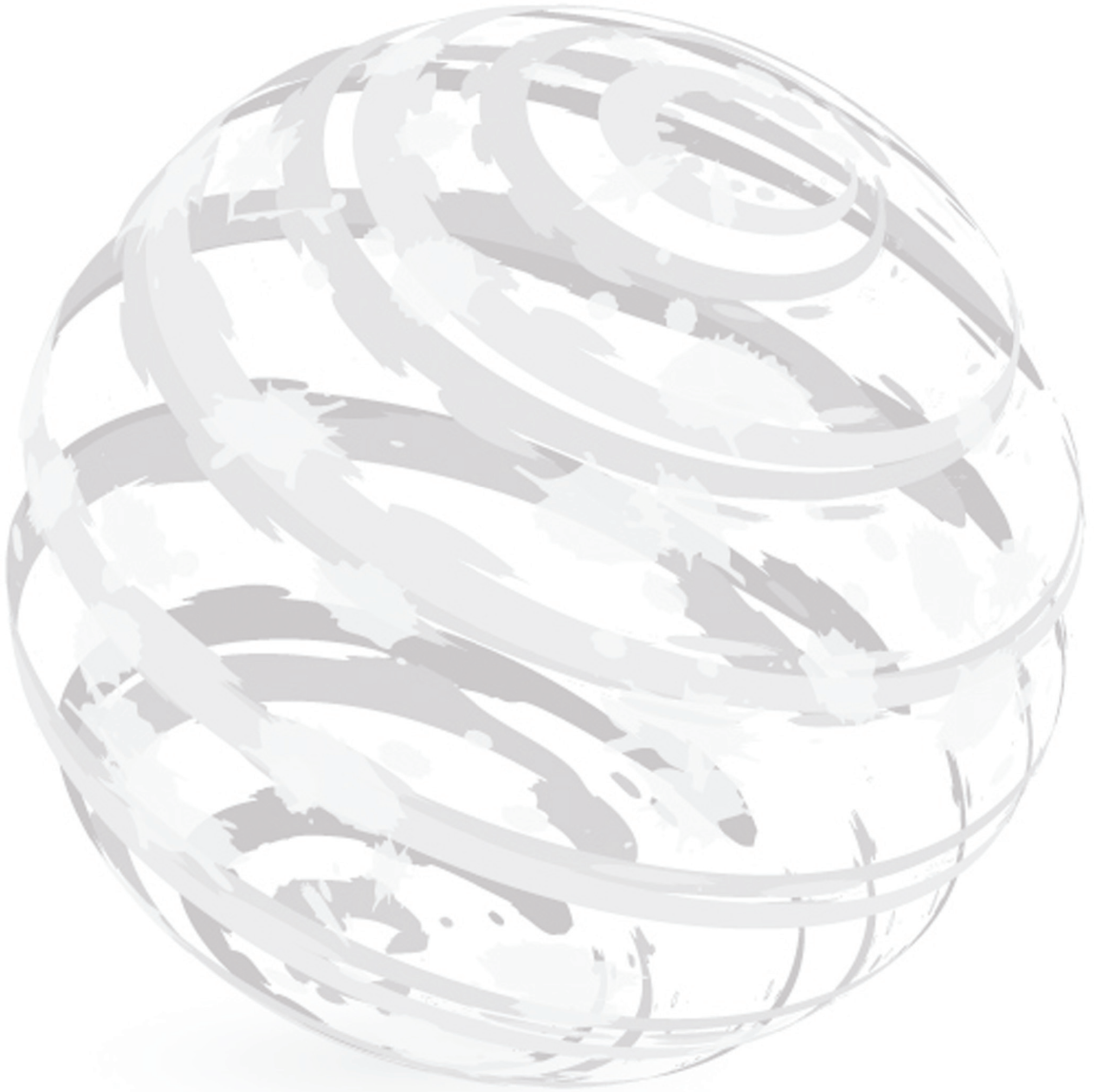

